\title{
Patterns of remnant discrete symmetries
}

\author{
Björn Petersen 1], Michael Ratz², Roland Schieren ${ }^{3}$ \\ Physik-Department T30, Technische Universität München, \\ James-Franck-Straße, 85748 Garching, Germany
}

\begin{abstract}
We analyze patterns of remnant discrete symmetries that arise from $\mathrm{U}(1)^{N}$ theories by spontaneous breaking. We describe a simple, geometrical way to understand these patterns and provide methods for identifying the discrete symmetries and bringing them to the simplest possible form. Applications in GUT and string model building are briefly discussed.
\end{abstract}

\footnotetext{
${ }^{1}$ Email: petersen@ph.tum.de

${ }^{2}$ Email: mratz@ph.tum.de

${ }^{3}$ Email: Roland.Schieren@ph.tum.de
} 


\section{Introduction}

Symmetries play a key role in our understanding of fundamental physics. While forces originate from continuous symmetries, discrete symmetries turn out to explain many important properties of matter. The perhaps most fundamental examples are the reflection and conjugation symmetries $P, T$ and $C$, yet there are other crucial $\mathbb{Z}_{N}$ symmetries. Examples for such symmetries include matter or $R$ parity in the minimal supersymmetric extension of the standard model (MSSM) [1]. Matter parity (or family reflection symmetry [2]) leads to a suppression of the proton decay rate and explains the stability of the MSSM dark matter candidate, the lightest supersymmetric particle (LSP). It can arise as a discrete $\mathbb{Z}_{2}$ subgroup of a baryon-minus-lepton-number symmetry $\mathrm{U}(1)_{B-L}$. One can break $\mathrm{U}(1)_{B-L}$ to matter parity by giving vacuum expectation values (VEVs) to fields with $B-L$ charge \pm 2 . In $\mathrm{SO}(10)$ grand unified theories (GUTs) one may switch on appropriate components of a $\overline{\mathbf{1 2 6}}$-plet (see e.g. [3]); in string theory an analogous breaking pattern can be achieved [4]. Discrete symmetries might also be an important ingredient for solving the flavor puzzle.

Obtaining discrete symmetries as remnants of gauge symmetries is, at a fundamental level, motivated by anomaly considerations. While discrete symmetries often are imposed ad hoc as global symmetries, it has been argued that global symmetries are violated by quantum effects unless they originate from a gauge symmetry via spontaneous symmetry breaking [5-7].

Here we focus on Abelian discrete symmetries i.e. $\mathbb{Z}_{N}$ groups. (For a recent discussion of how to obtain non-Abelian discrete symmetries by spontaneous breaking see [8].) It is well known how to obtain a single $\mathbb{Z}_{N}$-symmetry from a $\mathrm{U}(1)$; this will be reviewed below. On the other hand, the most promising candidate for a consistent theory of quantum gravity, string theory, typically provides us with models which exhibit a large rank gauge symmetry. This symmetry has to be (spontaneously) broken to the standard model in realistic vacua, which will generically lead to a non-trivial set of remnant discrete symmetries. The purpose of this study is to work out how such symmetries can be identified and put into a simple, i.e. canonical, form.

\section{Multiple $\mathbb{Z}_{N}$ symmetries from several $U(1)$ factors}

In this section we show how to determine the remnant symmetries if we break multiple $\mathrm{U}(1)$ factors by a set of VEVs. Throughout this study we shall assume the U(1) charges of all fields to be integers, which implicitly fixes our conventions for charge normalization. We start with the easiest example of one $\mathrm{U}(1)$ and two fields.

\subsection{Review: $\mathrm{U}(1) \rightarrow \mathbb{Z}_{q}$}

Consider a theory with gauge group $\mathrm{U}(1)$ and two complex scalar fields $\phi$ and $\psi$ (cf. [5]). Under the $\mathrm{U}(1)$ the fields transform according to

$$
\phi \rightarrow \mathrm{e}^{\mathrm{i} q \alpha(x)} \phi,
$$




$$
\psi \rightarrow \mathrm{e}^{-\mathrm{i} \alpha(x)} \psi
$$

with $q \in \mathbb{N}$, i.e. $\phi$ has charge $q$ and $\psi$ has charge -1 . The terms

$$
\phi^{*} \phi, \quad \psi^{*} \psi, \quad \phi^{*} \phi \psi^{*} \psi \text { and } \phi \psi^{q}+\text { h.c. }
$$

and powers as well as products thereof are gauge invariant. Suppose now that $\phi$ acquires a VEV. This leaves us with effective interaction terms of the form $\left(\psi^{q}\right)^{n}$ with $n \in \mathbb{N}$, dictated by the symmetry

$$
\psi \rightarrow \mathrm{e}^{2 \pi \mathrm{i} \ell / q} \psi \quad \text { with } \quad \ell=0,1, \ldots q-1 .
$$

An equivalent way to obtain this result is stating that the remaining symmetry is determined by the condition

$$
\mathrm{e}^{\mathrm{i} q \alpha(x)} \phi=\phi \Rightarrow q \alpha=2 \pi \ell \text { with } \quad \ell \in \mathbb{Z} .
$$

Hence,

$$
\frac{q \alpha}{2 \pi}=0 \bmod 1
$$

or, equivalently, $\alpha \in \frac{2 \pi}{q} \mathbb{Z}$. We have just rederived the well known result that, by giving a VEV to a field with charge $q$, the $\mathrm{U}(1)$ gets broken to a $\mathbb{Z}_{q}$ discrete subgroup. In what follows we will generalize this to situations in which several $U(1)$ s get broken to a number of $\mathbb{Z}_{n} \mathrm{~s}$.

\subsection{The general case}

Let us now consider the general case of a $\mathrm{U}(1)^{N}$ gauge theory with $M$ scalar fields $\phi^{(i)}$ $(1 \leq i \leq M)$, which will acquire VEVs, and $K$ other 'matter' fields $\psi^{(j)}(1 \leq j \leq K)$. We will denote the charge of the fields w.r.t. the $j^{\text {th }} \mathrm{U}(1)$ factor by $q_{j}\left(\phi^{(i)}\right)$ and $q_{j}\left(\psi^{(i)}\right)$ respectively. Accordingly, the $\phi^{(i)}$ fields transform as

$$
\phi^{(i)} \rightarrow \exp \left(\mathrm{i} \sum_{j} q_{j}\left(\phi^{(i)}\right) \alpha_{j}(x)\right) \phi^{(i)} .
$$

$q\left(\phi^{(i)}\right)$ can be thought of as an $N$-dimensional charge vector and $Q_{i j}=q_{j}\left(\phi^{(i)}\right)$ as an $M \times N$ charge matrix. Suppose now that $N>\operatorname{rank} Q$. In this case, there are unbroken $\mathrm{U}(1)$ factors. Then we can rotate the $\mathrm{U}(1)$ directions by an orthogonal transformation such that all $\phi^{(i)}$ will be uncharged under $(N-\operatorname{rank} Q) \mathrm{U}(1)$ factors. These U(1) factors will not be affected by the VEVs of the $\phi^{(i)}$ fields and we do not have to consider them any further. Therefore, without loss of generality, we will from now on consider the case $N \leq \operatorname{rank} Q$. Notice also that in supersymmetric theories the rank of the charge matrix cannot be maximal as $D$-flatness requires a non-trivial solution of $\sum_{i} n_{i} q\left(\phi^{(i)}\right)=0$ with $n_{i} \in \mathbb{N}_{0}$. 
To identify the remnant discrete symmetries after spontaneous symmetry breaking, consider the generalization of equation (4) in our simple example,

$$
\exp \left(\mathrm{i} \sum_{j} q_{j}\left(\phi^{(i)}\right) \alpha_{j}\right) \phi^{(i)} \stackrel{!}{=} \phi^{(i)} .
$$

This is equivalent to

$$
\sum_{j} q_{j}\left(\phi^{(i)}\right) \alpha_{j} \stackrel{!}{=} 2 \pi \ell^{i} \quad \text { with } \quad \ell^{i} \in \mathbb{Z}
$$

As in (4b), the right-hand side represents the usual 'mod conditions' for discrete breaking. Equation (6b) can be recast in matrix notation,

$$
Q \alpha \stackrel{!}{=} 2 \pi \ell \quad \text { with } \quad \ell \in \mathbb{Z}^{M}
$$

Recall that $Q$ is an $M \times N$ matrix with elements in $\mathbb{Z}$. Such a matrix can always be brought into diagonal form by two unimodular transformations, i.e. invertible matrices over $\mathbb{Z}$. Concretely, there exist $A \in \mathrm{GL}(M, \mathbb{Z})$ and $B \in \mathrm{GL}(N, \mathbb{Z})$ such that

$$
A Q B=D=\operatorname{diag}^{\prime}\left(d_{1}, \ldots, d_{N}\right) \quad \text { and } \quad d_{i} \text { divides } d_{i+1}
$$

Here diag' means that $D$ is an $M \times N$ matrix whose only non-zero elements $d_{i}$ are at the $i i$ positions. There are $N$ non-zero diagonal entries due to $\operatorname{rank} Q \geq N$. D is called Smith normal form (or just 'normal form' as in [9]) of $Q$. Note that unimodular matrices have two important properties:

1. They have determinant \pm 1 1

2. The greatest common divisor of all matrix elements in a single row is 1 because otherwise the determinant would not be \pm 1 . The same applies to each column.

Transformation (7) allows us to rewrite (6c),

$$
A^{-1} D B^{-1} \alpha=2 \pi \ell \text {. }
$$

Now multiply this equation by $A$. Due to the second property of unimodular matrices, the 'mod conditions' in equation (6c) remain unchanged, since $\ell^{\prime}=A \ell$ still takes all values in $\mathbb{Z}^{M}$ if $\ell$ does. Defining $\alpha^{\prime}=B^{-1} \alpha$ we arrive at

$$
\alpha_{j}^{\prime}=2 \pi \frac{\ell^{\prime j}}{d_{j}} \quad \text { with } \quad 0 \leq \ell^{\prime j} \leq d_{j}-1 .
$$

\footnotetext{
${ }^{1}$ If the determinant was zero, the matrices would not be invertible. If the absolute value of the determinant was greater than 1 , the inverse would not be an integer matrix, i.e. the matrix would not be invertible over $\mathbb{Z}$.
} 
Hence, we see that the remnant discrete symmetry is $\mathbb{Z}_{d_{1}} \times \ldots \times \mathbb{Z}_{d_{N}}$. If there are some $d_{i}=1$, the corresponding factors are trivial and can be omitted. The fields $\psi^{(j)}$ then transform according to

$$
\psi^{(j)} \rightarrow \exp \left(\mathrm{i} \sum_{k, \ell} q_{k}\left(\psi^{(j)}\right) B_{k \ell} \alpha_{\ell}^{\prime}\right) \psi^{(j)}=\exp \left(2 \pi \mathrm{i} \sum_{k} q_{k}^{\prime}\left(\psi^{(j)}\right) \frac{\ell^{\prime k}}{d_{k}}\right) \psi^{(j)}
$$

with new charges $q_{k}^{\prime}\left(\psi^{(j)}\right)=\sum_{i} q_{i}\left(\psi^{(j)}\right) B_{i k}$, which are defined modulo $d_{k}$. That is, we can choose $q_{k}^{\prime}\left(\psi^{(j)}\right) \in\left\{0, \ldots, d_{k}-1\right\}$.

\subsection{An example with two $\mathrm{U}(1)$ factors}

Let us illustrate the above procedure by an example. Consider a $\mathrm{U}(1) \times \mathrm{U}(1)^{\prime}$ theory with three fields obtaining VEVs and two other fields. That is, we have $N=2, M=3$ and $K=2$. The charges are given in table 1. In this example, we only consider scalar

(a) VEV fields.

$\begin{array}{ccc} & \mathrm{U}(1) & \mathrm{U}(1)^{\prime} \\ \phi^{(1)} & 8 & -2 \\ \phi^{(2)} & 4 & 2 \\ \phi^{(3)} & 2 & 4\end{array}$

(b) Matter fields.

$\begin{array}{ccc} & \mathrm{U}(1) & \mathrm{U}(1)^{\prime} \\ \psi^{(1)} & 1 & 3 \\ \psi^{(2)} & 1 & 5\end{array}$

Table 1: Charges of the fields with respect to the two U(1) factors.

fields, such that we do not have to worry about anomalies. Later, in the applications in section 5 we will discuss supersymmetric, anomaly-free settings. The charge matrix is given by the charges of the VEV fields (cf. table 1 (a)),

$$
Q=\left(\begin{array}{cc}
8 & -2 \\
4 & 2 \\
2 & 4
\end{array}\right)
$$

The diagonal matrix $D$ and the transformation matrix $B$ are

$$
D=\left(\begin{array}{ll}
2 & 0 \\
0 & 6 \\
0 & 0
\end{array}\right) \quad \text { and } \quad B=\left(\begin{array}{cc}
1 & -2 \\
0 & 1
\end{array}\right)
$$

Hence, we can read off that we are left with a $\mathbb{Z}_{2} \times \mathbb{Z}_{6}$ symmetry. The charges of the $\psi^{(i)}$ fields can be determined by multiplying their charge matrix by $B$ from the right,

$$
\left(\begin{array}{ll}
1 & 3 \\
1 & 5
\end{array}\right)\left(\begin{array}{cc}
1 & -2 \\
0 & 1
\end{array}\right)=\left(\begin{array}{ll}
1 & 1 \\
1 & 3
\end{array}\right)
$$

The new charges of the $\psi^{(i)}$ fields are given by the rows of this matrix. Altogether we find that the setting discussed here leads to a $\mathbb{Z}_{2} \times \mathbb{Z}_{6}$ symmetry, under which $\psi^{(1)}$ has charge $(1,1)$ and $\psi^{(2)}$ has charge $(1,3)$. 


\subsection{Visualization}

We will now provide a simple, geometrical way of envisaging the symmetry breaking patterns. First, notice that a direct product of groups $\mathbb{Z}_{d_{1}} \times \ldots \times \mathbb{Z}_{d_{N}}$ can be represented by an $N$-dimensional lattice. Each element of the group can be thought of as a point in the fundamental region or unit cell of the lattice. The volume of the unit cell is the number of elements and the order of the group.

Let us illustrate this by the above example. The VEV fields $\phi^{(i)}$ with charges as listed in table 1 (a) span the lattice. Since the first VEV field is an integer linear combination of the latter two, $\phi^{(1)}=3 \phi^{(2)}-2 \phi^{(3)}, \phi^{(2)}$ and $\phi^{(3)}$ span a basis of the charge lattice as illustrated by the arrows in figure 1(a). The matter fields $\psi^{(i)}$ are represented by the bullets. A coupling $\left(\psi^{(1)}\right)^{n_{1}}\left(\psi^{(2)}\right)^{n_{2}}$ is allowed by the discrete symmetries if and only if $n_{1} q\left(\psi^{(1)}\right)+n_{2} q\left(\psi^{(2)}\right)$ lies on a node in the charge lattice, which are represented by squares.

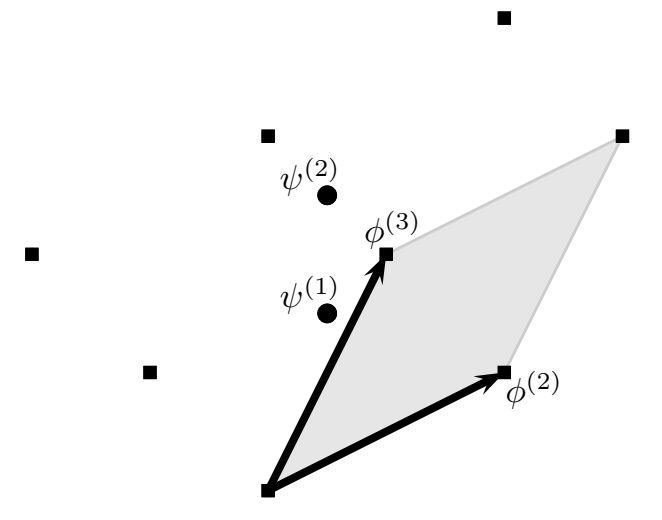

(a) Original lattice.

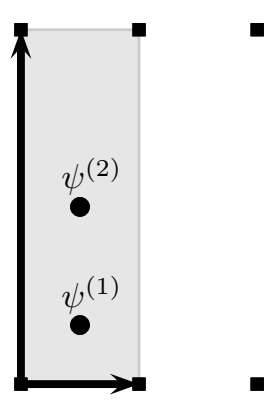

(b) Diagonalized lattice.

Figure 1: Illustration of discrete breaking.

Our procedure described in section 2.3 amounts to finding an orthogonal basis for the lattice which is given by the rows of the diagonal matrix $D$. The matrix $A$ performs a rotation of the $\phi^{(i)}$ charges which eliminates linear dependencies, while $B$ is the transformation between the bases. In the new basis, the lattice is orthogonal, and the $\psi^{(i)}$ charges are given by the projections on the basis vectors. We see that there is a $\mathbb{Z}_{2} \times \mathbb{Z}_{6}$ discrete symmetry where the $\mathbb{Z}_{2}$ corresponds to the horizontal and the $\mathbb{Z}_{6}$ to the vertical direction in figure $1(\mathrm{~b})$. The matter fields have charges $(1,1)$ and $(1,3)$, respectively, which are of course nothing but the coordinates of the bullets in figure 1(b). Note, however, that the true symmetry is smaller than that; we will describe in section 3 how to eliminate potential redundancies.

\subsection{Inverting the problem}

Often one is interested in 'inverting' the above procedure. Instead of determining a discrete symmetry that is left after certain fields attain VEVs, one would like to understand 
which fields need to be switched on in order to obtain a desirable discrete symmetry. Examples for such symmetries include matter parity and proton hexality [10]. We describe now a simple algorithm for accomplishing this task. We focus on the case of a remnant $\mathbb{Z}_{2}$ symmetry; the extension to the general case is straightforward.

(1) Start with a set 'matter' fields $\psi^{(i)}$ with charge vectors $q\left(\psi^{(i)}\right)$.

(2) The $q\left(\psi^{(i)}\right)$ span a lattice

$$
\Lambda=\left\{\sum_{\alpha} n_{\alpha} \lambda^{(\alpha)} ; n_{\alpha} \in \mathbb{Z}\right\}
$$

such that $q\left(\psi^{(i)}\right) \in \Lambda$, i.e.

$$
q\left(\psi^{(i)}\right)=\sum_{\alpha} n_{\alpha}^{(i)} \lambda^{(\alpha)} \quad \text { with } n_{\alpha}^{(i)} \in \mathbb{Z}
$$

and there is no coarser lattice with the same property 2

(3) Compute the dual lattice

$$
\Lambda=\left\{\sum_{\beta} n^{\beta} \lambda_{(\beta)}^{*} ; n^{\beta} \in \mathbb{Z}\right\}
$$

with $\lambda_{(\beta)}^{*} \cdot \lambda^{(\alpha)}=\delta_{\beta}^{\alpha}$. The basis vectors $\lambda_{(\beta)}^{*}$ have the obvious property that $\lambda_{(\beta)}^{*}$. $q\left(\psi^{(i)}\right)=n_{\beta}^{(i)} \in \mathbb{Z}$.

(4) Now try to build linear combinations

$$
\mathrm{t}_{\Delta}:=\sum_{\beta} m^{\beta} \lambda_{(\beta)}^{*}
$$

such that

$$
\mathrm{t}_{\Delta} \cdot q\left(\psi^{(i)}\right)=1 \bmod 2 .
$$

It is obvious that one just has to scan all possible combinations with $m^{\beta} \in\{0,1\}$ since an even $m^{\beta}$ will always lead to an even number on the right-hand side of (16). $\mathrm{t}_{\Delta}$ is then unique up to $\mathrm{U}(1)$ generators under which all $\psi^{(i)}$ are neutral.

(5) Given a generator $t_{\Delta}$, one has to check whether the model contains fields $\phi^{(i)}$ with charges of the type 'even over odd'. If this is the case, switching on the $\phi^{(i)}$ fields yields configurations with a $\mathbb{Z}_{2}$ symmetry under which the $\psi^{(i)}$ fields are odd.

Only the last two steps have to be slightly modified in order to obtain an arbitrary $\mathbb{Z}_{N}$ symmetry. In section 5.2 we will apply these methods in order to identify phenomenologically attractive string vacua.

\footnotetext{
${ }^{2}$ This lattice can, for instance, be obtained with the Mathematica command 'LatticeReduce'.
} 


\section{$3 \quad$ Simplifying multiple $\mathbb{Z}_{N}$ symmetries}

In this section we will consider a finite Abelian group $G=\mathbb{Z}_{d_{1}} \times \ldots \times \mathbb{Z}_{d_{N}}$ with $K$ fields $\psi^{(i)}, i=1, \ldots, K$, transforming under $G$. This setup may or may not be a result of the diagonalization procedure described in section $[2]^{3}$ Our aim is to eliminate redundancies, i.e. make the discrete symmetry 'as simple as possible'.

First, consider a toy example which consists of a $\mathbb{Z}_{6}$-symmetry with one field $\psi$ with charge 4 . This is equivalent to a $\mathbb{Z}_{3}$-symmetry where $\psi$ has charge 2 . In the case of

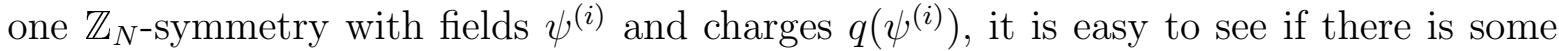
redundancy in our description of the symmetry. If the greatest common divisor (GCD) of the $q\left(\psi^{(i)}\right)$ and $N$ is greater than one, we can divide $q\left(\psi^{(i)}\right)$ and $N$ by their GCD. That is precisely what happened in our above toy example. This idea of reducing the group order carries over to the general case. In addition, the method we are going to develop will bring $G$ into a canonical form. Every finite Abelian group can be written as a direct product of the form

$$
G_{\text {canonical }}=\mathbb{Z}_{d_{1}} \times \ldots \times \mathbb{Z}_{d_{N}}, \quad \text { where } d_{i} \text { divides } d_{i+1} .
$$

The $d_{i}$ are uniquely determined by the group and are called invariant factors. The diagonalization process described in section 2 always leads to this form (cf. equation (17)).

\subsection{The general case}

Let us look at the charge matrix $\left(Q_{\psi}\right)_{i j}=q_{j}\left(\psi^{(i)}\right)$. This is a $K \times N$ matrix with integer entries. Again, the idea is to calculate the Smith normal form of $Q_{\psi}$. However, to get a meaningful result, we need to bring $G$ into the form $\mathbb{Z}_{d} \times \ldots \times \mathbb{Z}_{d}$ where $d$ is the least common multiple (LCM) of the $d_{i}$. This is because a $\mathbb{Z}_{d}^{N}$ discrete symmetry allows us to perform discrete rotations of the generators. Enlarging the symmetry implies a rescaling of the charge matrix, $\left(Q_{\psi}^{\prime}\right)_{i j}=\frac{d}{d_{j}} q_{j}\left(\psi^{(i)}\right)$. The fields transform now according to

$$
\psi^{(i)} \rightarrow \exp \left(2 \pi \mathrm{i} \sum_{j}\left(Q_{\psi}^{\prime}\right)_{i j} \alpha_{j}\right) \psi^{(i)} \quad \text { where } \quad \alpha_{j}=\frac{\ell_{j}}{d} \quad \text { with } \quad 0 \leq \ell_{j} \leq d-1 .
$$

$\left(Q_{\psi}^{\prime}\right)$ can be brought into Smith normal form $S$ by unimodular transformations $E \in$ $\mathrm{GL}(K, \mathbb{Z})$ and $F \in \mathrm{GL}(N, \mathbb{Z})$

$$
E Q_{\psi}^{\prime} F=S=\operatorname{diag}^{\prime}\left(s_{1}, \ldots, s_{k}\right) \quad \text { where } k=\min (K, N) .
$$

If $\operatorname{rank} Q_{\psi}^{\prime}<k$, some $s_{j}$ might vanish. The corresponding rows do not have to be considered further. This yields the transformation behavior

$$
\begin{aligned}
\psi^{(i)} & \rightarrow \exp \left(2 \pi \mathrm{i} \sum_{j, m, n} E_{i j}^{-1} S_{j m} F_{m n}^{-1} \alpha_{n}\right) \psi^{(i)} \\
& =\exp \left(2 \pi \mathrm{i} \sum_{j, n} E_{i j}^{-1} s_{j} F_{j n}^{-1} \frac{\ell_{n}}{d}\right) \psi^{(i)} .
\end{aligned}
$$

\footnotetext{
${ }^{3}$ That is, we do not require $d_{i} \mid d_{i+1}$ here.
} 
Just like in equation (9), we are allowed to define $\ell_{j}^{\prime}=\sum_{n} F_{j n}^{-1} \ell_{n}$. Hence, we get

$$
\psi^{(i)} \rightarrow \exp \left(2 \pi \mathrm{i} \sum_{j} E_{i j}^{-1} \frac{\ell_{j}^{\prime}}{d / s_{j}}\right) \psi^{(i)} .
$$

This tells us that we have rewritten our symmetry group $G$ as $\mathbb{Z}_{d_{1}^{\prime}} \times \ldots \times \mathbb{Z}_{d_{k}^{\prime}}$ where $d_{i}^{\prime}$ is the numerator of the (reduced) fraction $\frac{d}{s_{i}}$ and $d_{i+1}^{\prime}$ divides $d_{i}^{\prime}$. If a $d_{i}^{\prime}$ is equal to one, this factor can be omitted. The new charges of the fields, $q^{\prime \prime}\left(\psi^{(i)}\right)$, are encoded in the matrix $E^{-1}$,

$$
E^{-1}=\left(\begin{array}{c}
q^{\prime \prime}\left(\psi^{(1)}\right) \\
q^{\prime \prime}\left(\psi^{(2)}\right) \\
\vdots
\end{array}\right)
$$

These charges are equivalent to the the discrete charges $s_{i}^{\prime} q^{\prime}\left(\psi^{(i)}\right)$ (with $s_{i}^{\prime}$ denoting the denominator of the reduced fraction $d / s_{i}$ ), which one immediately reads off. An obvious consequence of our discussion is that after simplification there are at most as many $\mathbb{Z}_{d_{i}}$ factors as fields. Note that the volumes of the fundamental region of $G$ 's lattice $\left(V_{G}=\prod_{i=1}^{N} d_{i}\right)$ and the lattice spanned by the $\psi^{(i)}$ charges $\left(V_{\psi}=\operatorname{det} Q_{\psi}\right)$ provide a necessary but not sufficient criterion for redundancies: if both possess a GCD, the size of the symmetry may be reduced by this GCD.

\subsection{An alternative derivation}

Before discussing an example, let us present an alternative point of view. A coupling $\left(\psi^{(1)}\right)^{n_{1}} \cdots\left(\psi^{(K)}\right)^{n_{K}}$ is allowed by the discrete symmetry $G=\mathbb{Z}_{d_{1}} \times \ldots \times \mathbb{Z}_{d_{N}}$ only if there is a vector $n \in \mathbb{Z}^{K}$ such that

$$
Q_{\psi}^{T} n=\operatorname{diag}\left(d_{1}, \ldots, d_{N}\right) m
$$

with some $m \in \mathbb{Z}^{N}$ and $Q_{\psi}^{T}=\left(q\left(\psi^{(1)}\right), \ldots, q\left(\psi^{(K)}\right)\right)$. Equation (23) can be rewritten as

$$
\operatorname{diag}\left(\frac{d}{d_{1}}, \ldots, \frac{d}{d_{N}}\right) \cdot Q_{\psi}^{T} n=d m
$$

where $d$ denotes the LCM of the $d_{i}$, as before. Now we diagonalize the matrix on the left hand side of the equation,

$$
\operatorname{diag}\left(\frac{d}{d_{1}}, \ldots, \frac{d}{d_{N}}\right) \cdot Q_{\psi}^{T}=\left(F^{-1}\right)^{T} \cdot S \cdot\left(E^{-1}\right)^{T}
$$

with the $N \times K$ matrix $S=\operatorname{diag}^{\prime}\left(s_{1}, \ldots s_{k}\right)$, the unimodular matrices $E$ and $F$, and $k=\min (K, N)$, as before. Let $\nu$ denote the rank of $Q_{\psi}$, i.e. $S=\operatorname{diag}^{\prime}\left(s_{1}, \ldots s_{\nu}, 0, \ldots\right)$. Now (23) can be recast as

$$
\operatorname{diag}^{\prime}\left(\frac{s_{1}^{\prime}}{d_{1}^{\prime}}, \ldots, \frac{s_{\nu}^{\prime}}{d_{\nu}^{\prime}}, 0, \ldots\right)\left(E^{-1}\right)^{T} n=m^{\prime}
$$


where $m^{\prime}=F^{T} m$, and again $s_{i}^{\prime}$ and $d_{i}^{\prime}$ denote the numerators and denominators of the reduced fractions $s_{i} / d$, respectively. The rank of the matrix on the left-hand side of this equation is $\nu$. We can therefore truncate the equation,

$$
\operatorname{diag}\left(\frac{s_{1}^{\prime}}{d_{1}^{\prime}}, \ldots, \frac{s_{\nu}^{\prime}}{d_{\nu}^{\prime}}\right)\left(E_{\nu}^{-1}\right)^{T} n=m_{\nu}
$$

where $\left(E_{\nu}^{-1}\right)$ denotes the left $\nu$ columns of $E^{-1}$ (such that $\left(E_{\nu}^{-1}\right)^{T}$ is the upper $\nu \times K$ part of $\left.\left(E^{-1}\right)^{T}\right)$, and $m_{\nu} \in \mathbb{Z}^{\nu}$. This equation is equivalent to

$$
\left(E_{\nu}^{-1}\right)^{T} n=\operatorname{diag}^{\prime}\left(\frac{d_{1}^{\prime}}{s_{1}^{\prime}}, \ldots, \frac{d_{\nu}^{\prime}}{s_{\nu}^{\prime}}\right) m_{\nu}
$$

Comparing this equation with (23) reveals that the rows of $E_{\nu}^{-1}$ contain the charges of the $\psi^{(i)}$ and the $d_{i}^{\prime}$ determine the canonical symmetry.

\subsection{Example}

Let us continue the example of section 2.3. After breaking both $\mathrm{U}(1) \mathrm{s}$ we are left with a $\mathbb{Z}_{2} \times \mathbb{Z}_{6}$ and a charge assignment given in table 2 . We have to extend our symmetry
(a) Original
(b) 'Blown up'
(c) Minimal
charges.
charges.
charges.

$\begin{array}{cccccccc} & \mathbb{Z}_{2} & \mathbb{Z}_{6} & & \mathbb{Z}_{6} & \mathbb{Z}_{6} & & \mathbb{Z}_{6} \\ \psi^{(1)} & 1 & 1 & \psi^{(1)} & 3 & 1 & \psi^{(1)} & 1 \\ \psi^{(2)} & 1 & 3 & \psi^{(2)} & 3 & 3 & \psi^{(2)} & 3\end{array}$

Table 2: The example from section 2.3 continued. The original charges (a) are blown up to the charges of an extended $\mathbb{Z}_{6} \times \mathbb{Z}_{6}$ symmetry, which can be reduced to a $\mathbb{Z}_{6}$ symmetry by discrete rotations.

to $\mathbb{Z}_{6} \times \mathbb{Z}_{6}$. The charge matrix and the Smith normal form are

$$
Q_{\psi}^{\prime}=\left(\begin{array}{ll}
3 & 1 \\
3 & 3
\end{array}\right)=\left(\begin{array}{cc}
1 & 0 \\
3 & -1
\end{array}\right)\left(\begin{array}{ll}
1 & 0 \\
0 & 6
\end{array}\right)\left(\begin{array}{ll}
3 & 1 \\
1 & 0
\end{array}\right)=E^{-1} S F^{-1}
$$

The $d_{i}^{\prime}$ can be inferred from the diagonal matrix $S$ : they are given by 6 times the inverses of the diagonal entries, i.e. we have $d_{1}^{\prime}=6$ and $d_{2}^{\prime}=1$. The numerators are hence 6 and 1 , such that we are left with a $\mathbb{Z}_{6} \times \mathbb{Z}_{1}=\mathbb{Z}_{6}$ symmetry. The charges are given by the rows of $E^{-1}$ (modulo 6 ); since one factor is trivial we obtain that $\psi^{(1)}$ has charge 1 and $\psi^{(2)}$ has charge 3 . 


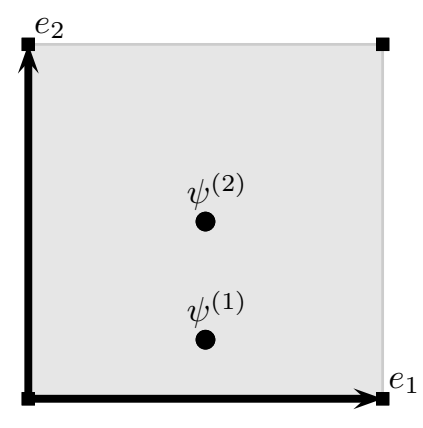

(a) Extended lattice.

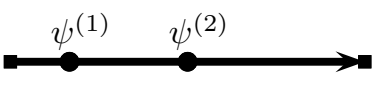

(b) Simplified lattice.

Figure 2: (a) Extended and (b) simplified charge lattices.

\subsection{Visualization}

The visualization works as before. We extend the lattice to $\mathbb{Z}_{6} \times \mathbb{Z}_{6}$ (figure 2 (a)). Our simplification process amounts to identifying a direction on which both nodes lie (figure 2 (b)). It is easy to see that $\psi^{(1)}$ sits at $1 / 6$ of the length of the one-dimensional $\mathbb{Z}_{6}$ lattice, corresponding to $\mathbb{Z}_{6}$ charge 1 , while $\psi^{(2)}$ sits at $1 / 2=3 / 6$ of the length, which leads to $\mathbb{Z}_{6}$ charge 3 .

\section{Automatization}

We provide a Mathematica package which automatically identifies the remnant symmetries, as discussed in section 2, and brings them to the canonical form, as described in section 3, on our web site [11]. This package has been used in our applications, which will be discussed in what follows.

\section{Applications}

\section{$5.1 \quad$ GUT model building}

Let us now apply the above methods to model building. We focus on a specific GUT model, which has been discussed in [12]. There, an anomaly-free $\mathbb{Z}_{6}$ symmetry was found that may allow us to suppress proton decay in $\mathrm{SO}(10)$ GUTs. The field content is given in table 3. There are three generations $\psi_{m}$ of standard model matter; $H$ and $H^{\prime}$

$\begin{array}{cccccccc} & \psi_{m} & H & H^{\prime} & \psi_{H} & \bar{\psi}_{H} & A & S \\ \mathrm{SO}(10) & \mathbf{1 6} & \mathbf{1 0} & \mathbf{1 0} & \mathbf{1 6} & \overline{\mathbf{1 6}} & \mathbf{4 5} & \mathbf{5 4} \\ \mathbb{Z}_{6} & 1 & -2 & 2 & -2 & 2 & 0 & 0\end{array}$

Table 3: Field content of an $\mathrm{SO}(10)$ model. 
contain the standard model Higgses. The other fields are used to break $\mathrm{SO}(10)$ down to the standard model gauge group $G_{\mathrm{SM}}$. Since $\mathrm{SO}(10)$ has rank 5 there is a $\mathrm{U}(1)$ factor, called $\mathrm{U}(1)_{\chi}$, in addition to the standard model gauge group. This $\mathrm{U}(1)_{\chi}$ gets broken by giving VEVs to the SM singlet fields contained in $\psi_{H}$ and $\bar{\psi}_{H}$, which have $\mathrm{U}(1)_{\chi}$ charges \pm 5 . Hence, we have a situation where a $U(1)_{\chi} \times \mathbb{Z}_{6}$ symmetry gets broken by a field with charges $( \pm 5, \pm 2)$. We can apply the routine described in section 2 . This can be accomplished by extending the $\mathbb{Z}_{6}$ to a $\mathrm{U}(1)$, introducing a dummy field with charges $(0,6)$ and assigning a VEV to this field.

We find that the $\mathrm{U}(1)_{\chi} \times \mathbb{Z}_{6}$ gets broken to a $\mathbb{Z}_{30}$ with charges given in table 4 . Let us

$\begin{array}{cccccccc} & Q & \bar{U} & \bar{D} & L & \bar{E} & H_{u} & H_{d} \\ \mathbb{Z}_{30} & 3 & 3 & 11 & 11 & 3 & 24 & 16 \\ \mathbb{Z}_{5} \times \mathbb{Z}_{6} & (1,1) & (1,1) & (2,5) & (2,5) & (1,1) & (3,4) & (2,0)\end{array}$

Table 4: The $\mathbb{Z}_{30} \simeq \mathbb{Z}_{5} \times \mathbb{Z}_{6}$ charges of the MSSM field content.

remark that the $\mathbb{Z}_{5}$ subgroup of this $\mathbb{Z}_{30}$ is redundant in the following sense: whenever a coupling is gauge invariant under $G_{\mathrm{SM}}$, the coupling is also invariant under $\mathbb{Z}_{5}$. This is because $\mathrm{SU}(5)$ has a non-trivial center $\mathbb{Z}_{5}$ (cf. the analogous discussion in [13]). Note also that $G_{\mathrm{SM}}$ invariance is the same as $\mathrm{SU}(5)$ invariance since the Cartan generators are equivalent. Our results imply that, if we assume that all states except the ones listed in table 4 attain masses, we are left with an anomalous field content. Specifically, the three generations of SM matter are anomaly free, but the $\mathbb{Z}_{6}$ charges of the Higgs fields exhibit an anomaly (for a discussion of discrete anomaly constraints see [14-16]). Using discrete anomaly matching [13] we can hence infer that the above assumption is inconsistent: either further light states have to be present, or the $\mathbb{Z}_{6}$ cannot be exact, i.e. we have to introduce further fields that attain VEVs. Of course approximate symmetry might be sufficient for suppressing the dangerous dimension five operators, and might well be correlated with flavor hierarchies (cf. the discussion in [17]). On the other hand, our findings show that the $\mathbb{Z}_{6}$ symmetry introduced in the $\mathrm{SO}(10)$ GUT in [12] cannot give rise to proton hexality [10].

\section{2 $\quad$ String model building}

As mentioned in the introduction, an exact matter parity can also be obtained in string theory by breaking a $\mathrm{U}(1)_{B-L}$ symmetry by two units $[4,18,19]$. This has led to a couple of vacuum configurations with an exact matter parity. Yet it turns out that, with this strategy, one is not always successful: within the so-called mini-landscape [18] of heterotic orbifolds with exact MSSM spectra, vacuum configurations with an exact matter parity could only be identified in a small fraction of the models; that is, an appropriate $\mathrm{U}(1)_{B-L}$ symmetry could only be identified in 15 out of 218 possible models. The obstacles encountered in this $\mathrm{U}(1)_{B-L}$-based approach are perhaps best illustrated in a concrete example. The model discussed in [20] (which later became absorbed in the mini-landscape) does have a $\mathrm{U}(1)_{B-L}$ symmetry $[19,21]$, yet in the $4 \mathrm{D}$ zero mode 
spectrum there is no field with an even $B-L$ charge (nor with a fractional charge of the type 'even over odd', which is also sufficient to provide us with matter parity [4]) 4

On the other hand, it is also clear that one does not really need a $U(1)_{B-L}$ symmetry with the standard charges for the MSSM matter fields. Any U(1) symmetry under which the matter states have odd charges, and for which there exist SM singlets with even charges, could do the job. Using the methods discussed in section 2.5, we were able to identify a collection of $G_{\mathrm{SM}}$ invariant fields $\phi^{(i)}$ that break the U(1) factors down to matter parity in the model presented in $[20,21]$. It is given by

$$
\begin{aligned}
\left\{\phi^{(i)}\right\}= & \left\{s_{1}, s_{2}, s_{3}, s_{5}, s_{7}, s_{9}, s_{12}, s_{14}, s_{16}, s_{18}, s_{19}, s_{20}, s_{22}, s_{23}, s_{24}, s_{34}, s_{39}, s_{40},\right. \\
& \left.s_{41}, s_{48}, s_{53}, s_{54}, s_{57}, s_{58}, s_{59}, s_{60}, s_{61}, s_{62}, s_{65}, s_{66}, f_{1-4}, \bar{f}_{1-4}, h_{1-14}\right\}
\end{aligned}
$$

in the notation of [21]. Our algorithm gives us a $\mathbb{Z}_{10}$ symmetry, however, as discussed in section 5.1, the $\mathbb{Z}_{5}$, which is just the non-trivial center of $\mathrm{SU}(5)$, is redundant. A quick scan indicates that in many (if not in all) mini-landscape models vacua with matter parity can be obtained. A detailed analysis of these issues and of the phenomenological properties of such vacua will be carried out elsewhere.

\section{Discussion}

We have described a simple method to determine symmetry breaking patterns which arise when $\mathrm{U}(1)^{N}$ gauge theories get broken to discrete subgroups. This method has a very simple geometrical interpretation: the fields acquiring VEVs define a charge lattice. Couplings $\left(\psi^{(1)}\right)^{n_{1}}\left(\psi^{(2)}\right)^{n_{2}} \ldots$ are only allowed by the remnant discrete symmetries if the sum of the charge vectors, $n_{1} q\left(\psi^{(1)}\right)+n_{2} q\left(\psi^{(2)}\right)+\ldots$, lies on a node of the charge lattice.

Unimodular transformations allow us to identify the remnant discrete symmetries, and to make them as simple as possible, i.e. to determine the true (or canonical) symmetries in a unique way.

We have applied our methods to model building. In the context of GUTs, we have identified an obstacle to completely forbidding dimension five proton decay operators in certain SO(10) GUTs. In string model building our methods allow us to identify novel vacuum configurations with an exact $R$ parity.

\section{Acknowledgements}

We would like to thank R. Kappl and P. Vaudrevange for discussions. This research is supported by the DFG cluster of excellence Origin and Structure of the Universe, the Graduiertenkolleg "Particle Physics at the Energy Frontier of New Phenomena" and the SFB-Transregios 27 "Neutrinos and Beyond" by the Deutsche Forschungsgemeinschaft

\footnotetext{
${ }^{4} \mathrm{In}$ [19], therefore, an alternative has been discussed where the $\mathrm{U}(1)_{B-L}$ gets broken by fields that do not appear in the $4 \mathrm{D}$ zero-mode spectrum but by states that are massless in an intermediate $6 \mathrm{D}$ orbifold GUT picture and get projected out by going to 4D. This requires a cancellation between a Kaluza-Klein mass and some non-trivial vacuum expectation values of certain standard model singlets.
} 
(DFG). Some parts of this work were carried out at the Aspen Center for Physics, which we would like to thank for the hospitality.

\section{References}

[1] G. R. Farrar and P. Fayet, Phys. Lett. B76 (1978), 575-579.

[2] S. Dimopoulos, S. Raby, and F. Wilczek, Phys. Lett. B112 (1982), 133.

[3] R. N. Mohapatra and P. B. Pal, World Sci. Lect. Notes Phys. 60 (1998), 1-397.

[4] O. Lebedev et al., Phys. Rev. D77 (2007), 046013, arXiv:0708.2691 [hep-th]].

[5] L. M. Krauss and F. Wilczek, Phys. Rev. Lett. 62 (1989), 1221.

[6] T. Banks, Nucl. Phys. B323 (1989), 90.

[7] J. Preskill and L. M. Krauss, Nucl. Phys. B341 (1990), 50-100.

[8] A. Adulpravitchai, A. Blum, and M. Lindner, (2009), 0907.2332.

[9] N. Jacobson, Basic Algebra I, W. H. Freeman and Company (1974), 471 p.

[10] H. K. Dreiner, C. Luhn, and M. Thormeier, Phys. Rev. D73 (2006), 075007, hep-ph/0512163.

[11] R. Schieren, DiscreteBreaking, http://einrichtungen.physik.tu-muenchen.de/T30e/codes/DiscreteBreaking/.

[12] R. N. Mohapatra and M. Ratz, Phys. Rev. D76 (2007), 095003, [0707.4070].

[13] C. Csáki and H. Murayama, Nucl. Phys. B515 (1998), 114-162, hep-th/9710105.

[14] L. E. Ibáñez and G. G. Ross, Phys. Lett. B260 (1991), 291-295.

[15] T. Banks and M. Dine, Phys. Rev. D45 (1992), 1424-1427, hep-th/9109045.

[16] T. Araki et al., Nucl. Phys. B805 (2008) 124 [arXiv:0805.0207 [hep-th]].

[17] A. T. Azatov and R. N. Mohapatra, Phys. Rev. D78 (2008), 015002, [0802.3906].

[18] O. Lebedev et al., Phys. Lett. B645 (2007), 88, [hep-th/0611095].

[19] W. Buchmüller and J. Schmidt, Nucl. Phys. B807 (2009), 265-289, [0807.1046].

[20] W. Buchmüller, K. Hamaguchi, O. Lebedev, and M. Ratz, Phys. Rev. Lett. 96 (2006), 121602, hep-ph/0511035.

[21] W. Buchmüller, K. Hamaguchi, O. Lebedev, and M. Ratz, Nucl. Phys. B785 (2007), 149-209, hep-th/0606187. 